\title{
Erysipelas-like presentation of Wells' syndrome (eosinophilic cellulitis)
}

\author{
N. Belfeki ${ }^{1}$, E. Gharbi ${ }^{1}$, C. Flateau ${ }^{2}$, S. Diamantis ${ }^{1}$ \\ ${ }^{1}$ Department of Internal Medicine; '2Department of Infectious Disease, \\ Groupe Hospitalier Sud lle de France, Melun, France
}

\section{SUMMARY}

Wells' syndrome, also called eosinophilic cellulitis, is a rare eosinophilic dermatosis characterized by an unspecific inflammatory erythematous eruption often associated with systemic symptoms. Here we report the case of a 57-year-old female with bilateral painful pitting and pruritic feet progressive for two weeks despite one week of oral antibiotics. Skin biopsy was performed showing dermal eosinophilic infiltration. The patient showed a spontaneous progressive improvement of the condition. The presented case demonstrates both clinical and histologic presence of lesions of Wells' syndrome in the course of the disease. A careful diagnostic approach is needed because of the lack of specific signs. The global outcome is favorable and spontaneous resolution is possible.

Key words: Wells' syndrome; eosinophilic cellulitis.

Reumatismo, 2019; 71 (4): 226-229

\section{INTRODUCTION}

W ells' syndrome, also called eosinophilic cellulitis, is a rare eosinophilic dermatosis characterized by an unspecific inflammatory erythematous eruption often associated with systemic symptoms. Histologically, the lesions are characterized by a superficial and deep-mixed inflammatory cell infiltrate containing eosinophils. It belongs to the large entity of eosinophilic dermatoses which can be idiopathic or associated with autoimmune diseases or hematological malignancy (1). Thus, a meticulous diagnostic approach is strongly needed to rule out alternative differential diagnoses. We report hereby the case of a female patient presenting with bilateral feet swelling and general symptoms, and illustrate the diagnosis challenge in such a rare situation.

\section{CASE REPORT}

A 57-year old female patient without past medical history was referred from the emergency room for bilateral painful pitting and pruritic feet lasting two weeks with prolonged fever, and joint pain which appeared later. She had previously received an ineffective course of oral antibiotics (amoxicillin $1 \mathrm{~g}$ t.i.d for 10 days) and denied any recent drug intake, travel, insect bite or allergy. Physical examination showed a high temperature of $39^{\circ} \mathrm{C}$, warm erythematous plaque of both feet associated with painful edema (Figure 1), swollen ankles and bilateral positive squeeze test. Other examinations showed normal cardiopulmonary auscul-

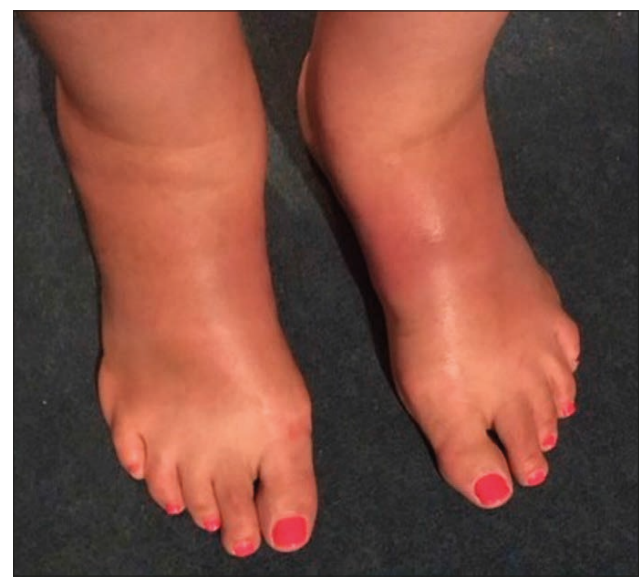

Figure 1 - Diffuse erythematous plaque of both feet. 


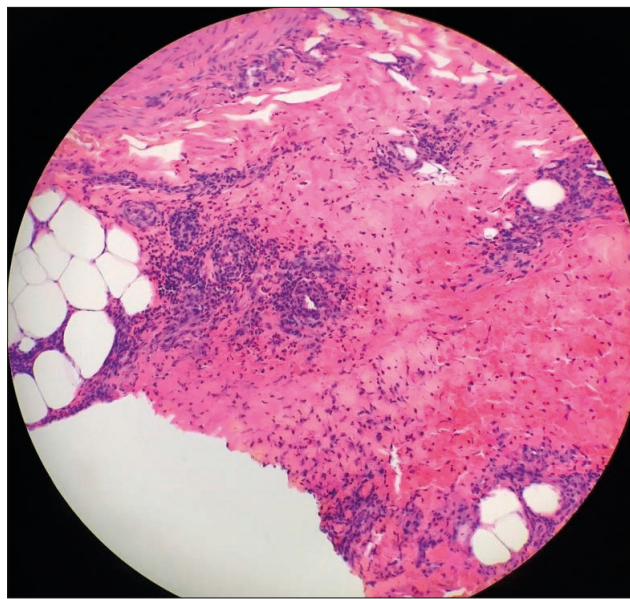

Figure 2 - Skin biopsy taken from the feet showing dermal edema and dermal infiltration by eosinophils consistent with Wells' syndrome. Hematoxylin and eosin stain.

tation, and neither lymph nodes enlargement nor hepatomegaly or splenomegaly. Routine biological investigation showed an inflammatory syndrome with elevated C reactive protein $(200 \mathrm{mg} / \mathrm{dL})$, normal cell blood count, normal electrolytes, creatinine and liver enzymes. Hepatitis $\mathrm{B}, \mathrm{C}$, and HIV serologies were negative. 24-h-proteinuria and urine test strips were normal. Anti-neutrophil cytoplasmic antibodies (ANCA), antinuclear antibodies (ANA), rheumatoid factors (RF), and anti-cyclic citrullinated peptides antibodies (ACPA) were negative. Thoracoabdominopelvic scanner was normal and feet MRI demonstrated swelling limited to the subcutaneous tissue. Skin biopsy showed edema in the dermis and infiltration of the dermis by eosinophils. No signs of vasculitis were shown. Tissue direct immunofluorescence was negative for $\operatorname{IgG}, \operatorname{IgA}$, IgM, IgE, and complement (Figure 2). Bacterial cellulitis was ruled out because of negative blood culture and an inefficient antibiotics course. Anamnesis did not find any past history of allergy or recent drug intake, there were no eosinophilia on CBC and Ig E level was normal. Thus, allergic contact dermatitis and/or drug reaction with eosinophilia and systemic symptom (DRESS) were excluded. Moreover, clinical and immunological investigations

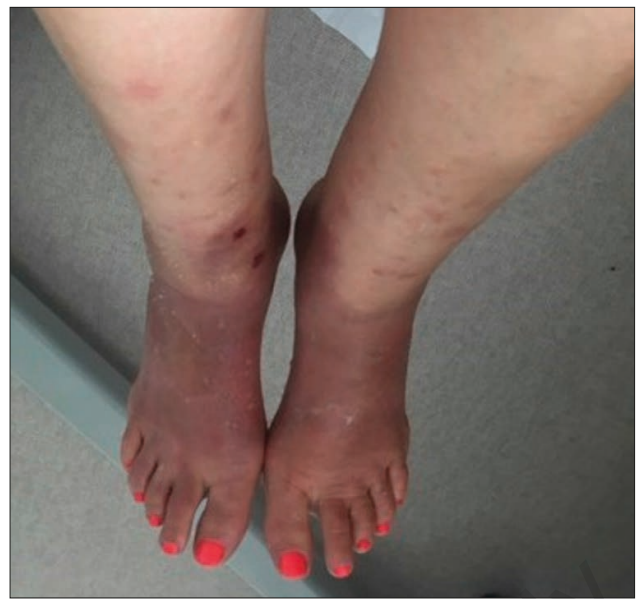

Figure 3 - Sclerodermiform-like evolution of both feet 4 weeks after diagnosis.

ruled out ANCA mediated vasculitis. Feet MRI did not show images of fasciitis and eosinophilic fasciitis (Shulman's fasciitis) was ruled out. The patient received symptomatic treatment. Paracetamol (1 g quid) for fever and joint pain and cetirizine (5 mg bid). She responded to bed rest. After one week in hospital, her temperature dropped while the joint pains and pruritus disappeared. The skin lesions healed progressively with at first a slight hyperpigmentation resembling systemic sclerosis (Figure 3) 4 weeks after discharge, which disappeared completely with ad integrum restitution three months later. At 12 months, clinical evaluation did not show relapse of cellulitis or symptoms and signs related to an associated autoimmune disease or malignancy.

\section{DISCUSSION AND CONCLUSIONS}

Wells' syndrome is a rare inflammatory skin condition presenting commonly with pruritic cellulitis-like plaques. There is a large polymorphism in the clinical features with the development of annular or circinate erythematous-edematous plaques or blistering, nodules, papulovesicular eruptions, and excoriated papules have been reported. In our case, the 
patient had a common presentation, with bilateral feet progressive cellulitis mimicking erysipela and absence of response to antibiotics. Anamnesis ruled out the hypothesis of insect bites, drug intake or allergic contact dermatitis that may mimic this presentation. Extra dermatological reported features were fever and inflammatory joints pain with markedly elevated inflammatory parameters. Throughout her course in hospital, the CT scan ruled out occult infection, hematological malignancy, or solid tumor. Moreover, feet MRI ruled out necrotizing fasciitis and showed swelling limited to the subcutaneous tissue. A skin biopsy was performed and pathological findings showed mild edema with dermal eosinophilic infiltration without signs of vasculitis. The absence of past history of asthma, polyneuropathy, abdominal pain, eosinophilia and negativity of anti-neutrophil cytoplasmic antibodies could rule out the hypothesis of eosinophilic granulomatosis with polyangiitis. The literature review (The PubMed and Ovid MEDLINE database Embase using the keywords eosinophilic cellulitis and Wells' syndrome) of histopathological findings revealed that dermal edema and eosinophilic infiltration were present in all cases and had to be considered as the gold standard for diagnosis of Wells' syndrome (1). Histopathological findings in Wells' syndrome varies between biopsy times, a fact that complicates interpretation. During the acute phase, we observe mainly dermic eosinophilic infiltration while during the subacute phase, flame figures are the hallmark picture. When chronic lesions are biopsied, we may have granuloma with giant cells $(2,3)$. The flame figures consist of eosinophilic major basic protein deposited on collagen bundles, and widespread degranulation of eosinophils is not to be considered as a pathognomonic histopathological indicator of Well's syndrome. They may be detected in other conditions such as bullous pemphigoid, eczema, prurigo, scabies, and drug eruption $(4,5)$. In our case, we did not find at the time of diagnosis or during follow-up a related underlying disease.
That is to say that Well's syndrome can be idiopathic. Neither did we reveal blood eosinophilia, which is reported in $50 \%$ of cases, and its level seems to be correlated to the severity of presentation (6). Once the diagnosis of Wells' syndrome is suspected, as based on clinical findings, it is corroborated by histopathological examination of a skin biopsy specimen. A careful diagnostic approach is needed to rule out differential diagnoses, which is a real challenge because of its rarity. Only 200 cases have been reported among published data (7). Based on the case reports reviewed, Wells' syndrome is often misdiagnosed and, thus, inappropriately treated. Many treatments have been used with variable success. It should be first noted that antibiotic therapy is characteristically ineffective. The most common and effective treatment is steroids (oral and/or topic). Antihistamines can be administered to relieve pruritus, but they are ineffective in clearing cutaneous lesions $(1,8)$. In case of relapses, ciclosporin or dapsone were effective when used alone and as an adjunct to systemic steroids to avoid the negative side effects of long-term highdose steroid use (9). In case of association with blood eosinophilia, mepolizumab (anti IL-5 monoclonal antibody) seems to be an interesting treatment to target option according to Busse et al. (9). We did not introduce steroids and treated the patient symptomatically. The patient recovered progressively and skin lesions disappeared progressively 3 months later. In fact, $12 \%$ of the reported cases showed a favorable spontaneous outcome (7). Further studies are needed to carify the best approaches to treatment.

\section{REFERENCES}

1. Sinno H, Lacroix JP, Lee J. Diagnosis and management of eosinophilic cellulitis (Wells' syndrome): A case series and literature review. Can J Plast Surg. 2012; 20: 91-7.

2. Moossavi M, Mehregan DR. Wells' syndrome: a clinical and histopathological review of seven cases. Int J Dermatol. 2003; 42: 62-7.

3. Peckruhn M, Tittelbach J, Schliemann S. Life of lesions in eosinophilic cellulitis (Wells' syn- 
drome) - a condition that may be missed at first sight. Am J Dermatopathol. 2015; 37: 15-7.

4. Aberer W, Konrad K, Wolff K. Wells' syndrome is a distinctive disease entity and not a histologic diagnosis. J Am Acad Dermatol. 1988; 18: 105-14.

5. Wood C, Miller AC, Jacobs A. Eosinophilic infiltration with flame figures. A distinctive tissue reaction seen in Wells' syndrome and other diseases. Am J Dermatopathol. 1986; 8: 186-93.

6. Espana A, Sanz ML, Sola J. Wells' syndrome (eosinophilic cellulitis): correlation between clinical activity, eosinophil levels, eosinophil cation protein and interleukin-5. Br J Dermatol. 1999; 140: 127-30.

7. Muller T, Baubion E, Amazan E. Well's cellulitis: A case report. Rev Med Interne. 2017; 38: 407-411.

8. Lee MW, Nixon RL. Eosinophilic cellulitis case report: Treatment options. Australas J Dermatol. 1994; 35: 95-7.

9. Busse WW, Ring J, Huss-Marp J. A review of treatment with mepolizumab, an anti-IL-5 mAb, in hypereosinophilic syndromes and asthma. J Allergy Clin Immunol. 2010; 125: 803-13. 